\title{
Vivência de estudantes de enfermagem como monitores na disciplina de semiologia e Semiotécnica: relato de experiência
}

\author{
Experience of nursing students as monitors in semiology and Semitechnical discipline: \\ experience report
}

Experiencia de estudiantes de enfermería como monitores en la Semiología y la disciplina semiotéctica: informe de experiência

Jéssica Carvalho Guimarães ${ }^{1}$, Laís Gomes dos Santos ${ }^{1 *}$, Andriele Valentim da Costa ${ }^{1}$, Luana de Melo Lisboa ${ }^{1}$, Valdenora Patrícia Rodrigues Macedo².

\section{RESUMO}

Objetivo: Relatar a experiência de estudantes de enfermagem no Programa de Monitoria, oferecido por uma universidade pública no interior do Amazonas. Métodos: Trata-se de um relato de experiência de discentes do curso de Enfermagem, inseridas no Programa de Monitoria, ocorrido no primeiro semestre de 2017, na disciplina de Semiologia e Semiotécnica. Relato da experiência: As monitoras assumem responsabilidades que as aproxima da docência, por isso, desempenharam as atribuições com garra e determinação, embora tenham vivenciado algumas dificuldades ao longo do percurso, tais como: escassez de materiais, falta de protocolos institucionais dos procedimentos realizados e, a incompatibilidade de horários para cumprir adequadamente a monitoria. Considerações finais: A monitoria proporcionou elo entre professor-monitormonitorado, onde foi possível perceber as dificuldades enfrentadas pelos monitores, principalmente, por conta da existência de um quantitativo de alunos superior ao suportado pelo laboratório, acarretando prejuízo no desenvolvimento das atividades, dificultando a melhor atuação dos monitores. Contudo sugere-se a implantação de protocolos dos procedimentos realizados nos laboratórios, bem como, planejamento na distribuição das disciplinas otimizando assim horários compatíveis para melhor exercer o papel de monitor.

Palavras-chave: Enfermagem, Ensino, Laboratórios, Estudante.

\begin{abstract}
Objective: To report the experience of nursing students in the Monitoring Program, offered by a public university in the interior of Amazonas. Methods: This is an experience report of Nursing students, inserted in the Monitoring Program, which took place in the first semester of 2017, in the Semiology and Semiotics discipline. Experience report: The monitors assume responsibilities that bring them closer to teaching, so they performed the duties with determination and determination, although they experienced some difficulties along the way, such as: lack of materials, lack of institutional protocols of the procedures performed and , mismatch of times to properly comply with monitoring. Final considerations: Monitoring provided a link between teacher-monitor-monitor, where it was possible to perceive the difficulties faced by the monitors, mainly due to the existence of a larger number of students than the laboratory, causing impairment in the development of activities, hindering the best performance of the students. monitors. However, it is suggested the implementation of protocols of the procedures performed in the laboratories, as well as planning the distribution of disciplines thus optimizing compatible schedules to better exercise the role of monitor.
\end{abstract}

Keywords: Nursing, Teaching, Laboratories, Student.

\section{RESUMEN}

Objetivo: Reportar la experiencia de los estudiantes de enfermería en el Programa de Monitoreo, ofrecido por una universidad pública en el interior de Amazonas. Métodos: Este es un informe de experiencia de estudiantes de enfermería, insertado en el Programa de Monitoreo, que tuvo lugar en el primer semestre de 2017, en la disciplina de Semiología y semiótica. Informe de experiencia: Los monitores asumen responsabilidades que los acercan a la enseñanza, por lo que desempeñaron los deberes con determinación,

${ }^{1}$ Universidade Federal do Amazonas (UFAM), Coari - AM. *E-mail: laisgomes384@gmail.com 
aunque experimentaron algunas dificultades en el camino, tales como: falta de materiales, falta de protocolos institucionales de los procedimientos realizados y, desajuste de tiempos para cumplir adecuadamente con el seguimiento. Consideraciones finales: El monitoreo proporcionó un vínculo entre maestro-monitor-monitor, donde fue posible percibir las dificultades que enfrentan los monitores, principalmente debido a la existencia de un mayor número de estudiantes que el laboratorio apoyado, lo que causó un deterioro en el desarrollo de las actividades, lo que obstaculizó el mejor desempeño de los estudiantes. Monitores sin embargo, se sugiere la implementación de protocolos de los procedimientos realizados en los laboratorios, así como la planificación de la distribución de disciplinas, optimizando así los horarios compatibles para ejercer mejor el papel del monitor.

Palabras clave: Enfermería, Docencia, Laboratorios, Estudiante.

\section{INTRODUÇÃO}

O programa de monitoria da Universidade Federal do Amazonas UFAM), regulamentado pela Resolução no 006/2013, tem por objetivo, iniciar e estimular a participação de alunos de graduação nas diversas atividades docentes de nível superior, contribuindo assim, com o processo de ensino-aprendizagem, na relação docente-discente e discente-discente, buscando maior interação e obtenção do aprendizado, com aproveitamento satisfatório nas disciplinas (RESOLUÇÃO, 006/2013).

Dentre as atribuições que compete ao monitor destacam-se: Constituir elo entre professor e estudantes da disciplina, visando o desenvolvimento da aprendizagem; Apoiar o professor na realização e orientação de trabalhos práticos e experimentais, na preparação de material didático e em atividades da disciplina, em sala de aula, em laboratório e em campo, bem como, a produção técnica cientifica relativa às atividades de monitoria; Participar de atividades que propicie o aprofundamento na disciplina e cumprir doze horas semanais de atividades de monitoria (RESOLUÇÃO, 006/2013).

Diante das atividades previstas nesta resolução é possível notar que o estudante auxilia o professor em atividades cotidianas bastante significativa em todas as etapas de ensino, proporcionando a ampliação do conhecimento, estimulando aptidões e aprimorando habilidades no campo de ensino, justificando assim, a importância da monitoria e os benefícios que a mesma oferece para os estudantes, professores e instituição de ensino (RESOLUÇÃO, 006/2013).

Andrade EGR, et al. (2018) descreve o ensino-aprendizagem como um processo no qual deve haver diálogo entre discente e docente, desta forma, destacando a monitoria acadêmica como fortalecedora desse processo e evidenciando a importância da monitoria no meio acadêmico, uma vez que o torna uma referência estudantil por sua postura ética, respeitosa e suas atitudes exemplares no compartilhamento do saber.

A associação da disciplina de Semiologia e Semiotécnica no currículo do curso de Enfermagem tem seu papel fundamental para a compressão da construção e preparação do estudante de enfermagem, pois ela abrange e relaciona o conteúdo teórico e prático da profissão (MELO GSM, et al., 2016).

De acordo com Melo GSM, et al. (2016) semiologia é a investigação e o estudo de sinais e sintomas que o cliente pode apresentar durante a anamnese e, Semiotécnica abrange o estudo e os métodos utilizados como as ações necessárias que sucederão ao exame físico.

As atividades de monitoria são dificultadas por fatores como o desconhecimento dos alunos quanto às normas de biossegurança e institucionais vigentes para a utilização dos laboratórios, sendo necessária uma organização prévia, o que exige comprometimento e responsabilidade e possibilita satisfação pelas contribuições significativas no processo de formação acadêmica dos alunos monitorados (CARVALHO IS, et al., 2012).

No processo de ensino-aprendizagem, espera-se que os estudantes ao adentrarem nas unidades de saúde para desenvolverem aulas práticas, acompanhadas por professores enfermeiros estejam munidos de conhecimento teórico-prático adquirido previamente, pois existe quase sempre uma grande oportunidade deles executarem os procedimentos de competência do enfermeiro sob supervisão direta do preceptor, afinal, 
é no campo de aulas práticas que as destrezas são colocadas em práticas e as habilidades aperfeiçoadas, exigindo alto nível de conhecimento para a aquisição das competências atribuídas ao estudante (ANDRADE EGR, et al, 2018).

A monitoria contribui de forma significativa para o aprendizado dos alunos envolvidos, possibilitando a troca de experiências, compartilhamento dos saberes, fortalecendo vínculos entre alunos e professores, adquirindo responsabilidades, gerenciando problemas e novos desafios com conhecimento, autonomia e trabalho em equipe, proporcionando uma relação interpessoal harmoniosa baseado em ética e respeito (MATOSO, 2014).

Percebe-se que o programa de monitoria é fundamental para a construção do conhecimento. Desse modo, o presente estudo tem como objetivo relatar a experiência de estudantes de enfermagem enquanto monitores na disciplina de semiologia e Semiotécnica da enfermagem, considerando que esta disciplina é a base para o desenvolvimento das demais atividades e habilidades dos futuros profissionais enfermeiros.

\section{METODOLOGIA}

Trata-se de um relato de experiência, descrito sobre atuação de discentes do curso de Enfermagem, que estiveram inseridas no Programa de Monitoria, oferecido por uma universidade pública, localizado na capital e interior do estado do Amazonas. Ocorrido no primeiro semestre de 2017, na disciplina de Semiologia e Semiotécnica, contemplada com carga horária de 180 horas, entre teórico-prático, ofertada na ocasião para 43 discentes do quarto período do curso de Enfermagem.

As atividades de monitoria foram desenvolvidas em duas etapas: na primeira, o professor coordenador da monitoria e monitores reuniam-se para discutir e planejar as atividades desenvolvidas no Laboratório de Enfermagem, assim como, os monitores destinavam-se a estudar para aperfeiçoar seus conhecimentos. $\mathrm{Na}$ segunda, os monitores realizavam as atividades de orientação dos procedimentos de enfermagem após o conteúdo teórico dado em sala de aula pelo professor e posteriormente, era ministrado aula prática no laboratório do assunto pelo mesmo professor de maneira planejada. Ainda nesta fase, os monitores tiveram a oportunidade de planejar e executar simulação de prova prática laboratorial ao longo do semestre, cumprindo carga horária de 12 horas semanais de forma voluntária. É importante ressaltar que o presente relato respeitou os rigores éticos no que tange o ensino-aprendizagem no contexto universitário.

\section{RELATO DA EXPERIÊNCIA}

A monitoria de Semiologia e Semiotécnica da Enfermagem foi desenvolvida dentro e fora do laboratório de enfermagem com planejamento e organização das atividades realizadas de segunda a sexta-feira, nos turnos matutino e/ou vespertino de acordo com cada conteúdo programado, semanalmente.

Visando a aproximação dos acadêmicos monitores e demais discentes, as atividades tiveram início com a apresentação dos monitores da disciplina, do cronograma de atendimento dos mesmos e as normas do laboratório de enfermagem, que consistiam em alertar para utilização da vestimenta recomendada no ambiente laboratorial, tais como: calça comprida, sapatos fechados, jalecos e cabelos presos. Carmo IC, et al. (2016) destaca a utilização de equipamentos de segurança para a prevenção de acidentes dentro dos laboratórios e preparando melhor os estudantes para o ambiente hospitalar, uma vez que os riscos de acidentes com materiais perfurocortantes e biológicos são evidentes.

Com intuito de aperfeiçoar as orientações realizadas pelos monitores aos estudantes, observaram-se primeiramente as características locais do laboratório, onde foi percebido que a estrutura do laboratório não era compatível com o contingente de pessoas que usariam. Mediante a isto, foi necessário dividir a turma em dois subgrupos, cujo objetivo era de proporcionar melhor dispêndio de atenção durante as atividades programadas.

Brito FMM, et al. (2018) traz a reflexão de que este espaço favorece a formação crítica, sendo necessário que o mesmo seja visto para além de seus aspectos instrumentais, favorecendo e possibilitando o 
desenvolvimento das habilidades técnicas. A prática simulada é mais do que uma tentativa de treino e replicação de um contexto ou situação ideal, é possibilidade de analisar o real.

No que se refere à primeira etapa do percurso da monitoria, pode-se destacar as reuniões periódicas realizadas pelo professor orientador aos monitores. Elas foram de fundamental importância, pois, eram realizadas de modo a estimular os monitores não somente para o aperfeiçoamento das técnicas, mas, para revisar as literaturas indicadas e proporcionar um elo de ligação.

Deste modo, atuaram como mediadores no levantamento de pesquisas atualizadas sobre os assuntos que seriam explanados ao longo do semestre, também serviam de momentos para que as demonstrações dos procedimentos fossem repassadas e praticadas igualmente, onde as dúvidas fossem sanadas.

Na segunda fase do percurso, os monitores ficavam responsáveis em preparar o laboratório para as aulas programadas, com os materiais e equipamentos necessários para as práticas dos procedimentos, de acordo com o assunto que havia sido ministrado previamente.

O papel dos monitores com os monitorados era de subsidiar o aporte dos procedimentos teórico-prático, ou seja, assessoravam e supervisionavam o desenvolvimento do estudante na disciplina, contribuindo com o desenvolvimento das habilidades e ganho de competências para o bom desenvolvimento das atividades em campo de aula prática hospitalar. No momento da monitoria, os monitores apontavam aspectos positivos e fortaleciam os vistos fragilizados nos estudantes.

Essa observação dos monitorados é confirmada com a melhoria no desenvolvimento das técnicas e por demonstração de novas habilidades. Carvalho IS, et al. (2012) afirma que a monitoria cria uma experiência diferenciada, através da consolidação dos conhecimentos teórico-práticos; segurança quanto à realização de procedimentos inerentes a disciplina e maior visibilidade acadêmica; aprofundamento de relações com discentes dos demais períodos do curso e com os professores da disciplina; e visão crítica acerca do processo de ensino e aprendizagem, apontam a contribuição do programa de monitoria como meio relevante para melhoria do aprendizado.

A experiência aproximou os monitores da docência, sendo este, um dos objetivos do programa, que culminaram na elaboração e programação das aulas práticas, orientações individuais, o rigor no cumprimento dos horários de aulas, a estimulação pela busca dos conhecimentos, incentivo à pesquisa, extensão entre outros. Essas atividades agregaram valores inestimáveis na vida acadêmica, onde foi gratificante atua.

Os assuntos ministrados em sala de aula e, posteriormente, demonstrados no laboratório de enfermagem eram sempre ensinados pelos professores e após com a participação dos monitores para o desenvolvimento individual e coletivo dos monitorados, tais procedimentos estão descritos no (Quadro 1).

Quadro 1 - Procedimentos realizados no laboratório de enfermagem na disciplina de semiologia e Semiotécnica, no decorrer da monitoria em 2017.

\begin{tabular}{|c|l|}
\hline $\begin{array}{c}\text { Medidas de } \\
\text { biossegurança }\end{array}$ & - Lavagem das mãos; \\
\hline & - Utilização de equipamentos de proteção individual (EPI's) \\
\hline & - Diluição, cálculo e administração de medicamentos; \\
& - Aferição de sinais vitais; \\
& Exame físico cabeça, pescoço, fâneros e anexos, sistema cardiovascular, \\
Procedimentos & - Aplicação de enteroclisma; \\
& - Passagem de cateter nasogástrica, nasoenteral, vesical de demora e de \\
& alívio masculina e feminina; \\
& - Mudança de decúbito, transporte de paciente, restrição no leito, \\
& - Bovimentação passiva e ativa; \\
& - Limpo no leito, higiene íntima masculina e feminina; \\
\hline
\end{tabular}

Fonte: GUIMARÃES JC, SANTOS LG, COSTA AV, et al., 2019. 
Ressalta-se que ao longo das atividades os monitores sentiram algumas dificuldades. Entre tais, pontuase a escassez de materiais, falta de protocolos institucionais dos procedimentos realizados, literaturas desatualizadas, incompatibilidade de horários para cumprir adequadamente a monitoria.

A falta de matérias configurou um dos grandes problemas, afetando a demonstração dos procedimentos corretamente, o que nos chamou a atenção pela necessidade de adequação do cenário local devido à repercussão negativa por parte dos monitorados ao serem observados durante a execução dos procedimentos, surgindo a necessidade, algumas vezes, de improvisos para executá-las.

No que tange a literatura, percebeu-se divergência ao revisar o conteúdo da passagem de cateter vesical de demora. $\mathrm{O}$ que serviu de alerta para discussão com professores e demais monitores, percebendo a necessidade da criação de protocolos estabelecidos no instituto.

Os protocolos facilitam a compreensão para a realidade local, o que contribui com o aprendizado, uma vez que não há disponibilidade de livros suficientes para todos os estudantes e a falta de consenso entre literaturas é notório. Assim como, adequar as disciplinas com o intuito de disponibilizar horários para a oferta das monitorias, para que não haja incompatibilidade de horários e os monitores não consigam exercer o seu papel, prejudicando assim os monitorados.

Aos monitores foi dada a oportunidade de organizarem um simulado dos principais procedimentos desenvolvidos na monitoria, tais como: lavagem das mãos, calçamento de luvas, cálculo e administração de medicamentos intramuscular e endovenosa, exame físico cardiopulmonar e gastrointestinal, lavagem intestinal, cateter nasogástrica e nasoenteral, técnica de curativo e sondagem vesical de demora masculina e feminina, que compuseram a prova prática.

No simulado os monitores organizaram o espaço, colocaram em prática a capacidade administrativa de prevê e prover materiais e equipamentos para o dia da prova oficial. Sem dúvida a realização do simulado, bem como, o auxílio dos monitores na prova prática configurou-se um aprendizado valioso no processo do aprendizado sobre o campo da docência para os monitores. Ao entrarem na sala, os discentes realizavam o primeiro passo que consistia no sorteio de três procedimentos, em seguida eram acompanhados pelos docentes e monitores ao realizarem os temas sorteados. Na sua aplicabilidade, a avaliação contemplava os conhecimentos teórico-prático relacionado ao domínio e destreza da técnica.

$\mathrm{Na}$ ocasião foi possível perceber o quão fundamental é o programa oferecido aos estudantes dentro de uma instituição, considerando a contribuição positiva proporcionada a todos os estudantes, porém, pode-se observar uma baixa adesão no período citado da monitoria. Prevalecendo com maior destaque, aqueles com maior frequência nos dias e hora agendada para atendimentos junto aos monitores, ou seja, foi evidente nesta monitoria uma baixa procura do serviço ao longo do semestre, porém o cenário mudava, quando antecedia as provas práticas.

Desta forma, nota-se de fundamental importância à atuação dos monitores na disciplina de semiologia e Semiotécnica, tendo em vista uma carga horária alta, exigindo do professor o auxílio de pessoas com habilidades e competências, capacitadas para conduzir a execução dos procedimentos, principalmente no laboratório de Enfermagem que requer disponibilidade de carga horária para dispor aos monitorados poderem treinar.

\section{CONSIDERAÇÕES FINAIS}

Esta vivência nos proporcionou uma experiência impar em quanto acadêmicas, nos mostrando que o papel do monitor se faz necessário, pois o mesmo apresenta uma maior permeabilidade para diálogo com os discentes, se tornando um facilitador no processo de ensino/aprendizagem, podendo compartilhar com os discentes suas experiências e estimulá-los a prática da monitoria na vida acadêmica. Embora a monitoria tenha sido um elo entre professor-monitor-monitorado, visando o ensino aprendizado no papel de monitor, foi possível perceber as dificuldades enfrentadas pelos professores, principalmente, por conta da existência de um quantitativo de alunos, superior ao suportado pelo laboratório, acarretando prejuízo no desenvolvimento 
das atividades, dificultando a melhor atuação dos monitores. Contudo sugere-se a implantação de protocolos dos procedimentos realizados nos laboratórios, bem como, planejamento na distribuição das disciplinas otimizando, assim horários compatíveis para melhor exercer o papel de monitor.

\section{REFERÊNCIAS}

1. ANDRADE EGR, et al. Contribuição da monitoria acadêmica para o processo ensino-aprendizagem na graduação em enfermagem. Revista brasileira da enfermagem, Brasília, v.71, 2018.

2. BRITO FMM, et al. Laboratório de enfermagem e a formação crítica de enfermeiros: aproximações e distanciamentos. Revista brasileira da enfermagem, Brasília, v.71, 2018.

3. CARVALHO IS, et al. Monitoria em semiologia e Semiotécnica para a enfermagem: um relato de experiência. Revista de Enfermagem da UFSM, Rio Grande do Sul, 2012.

4. CARMO IC, et al. Segurança e enfermagem: reflexões sobre o ensino da biossegurança nos cursos de enfermagem. Revista de Educação, Ciência e Tecnologia do IFRS. Porto Alegre, v.3, n.2, 2016.

5. MATOSO, LML. A importância da monitoria na formação acadêmica do monitor: um relato de experiência. Revista científica da escola da saúde,

6. MELO GSM, et al. Semiologia e Semiotécnica da enfermagem: avaliação dos conhecimentos de graduandos sobre procedimentos. Revista brasileira da enfermagem, Brasília, v. 70, n. 2, 2016.

7. RESOLUÇÃO № 006/2013. Universidade Federal do Amazonas. Regulamenta o Programa de Monitoria da Universidade Federal do Amazonas. Manaus, 2013. 